\title{
Introduction of gelatin microspheres into an injectable calcium phosphate cement
}

\author{
W. J. E. M. Habraken, ${ }^{1}$ L. T. de Jonge, ${ }^{1}$ J. G. C. Wolke, ${ }^{1}$ L. Yubao, ${ }^{2}$ A. G. Mikos, ${ }^{3}$ J. A. Jansen ${ }^{1}$ \\ ${ }^{1}$ Department of Periodontology and Biomaterials, Radboud University Nijmegen Medical Center, Nijmegen, \\ The Netherlands \\ ${ }^{2}$ Department of Nano-biomaterials Center, Analytical and Testing Center, Sichuan University, Chengdu 610064, \\ People's Republic of China \\ ${ }^{3}$ Department of Bioengineering, Rice University, Houston, Texas 77251
}

Received 4 April 2007; revised 3 July 2007; accepted 31 July 2007

Published online 11 January 2008 in Wiley InterScience (www.interscience.wiley.com). DOI: 10.1002/jbm.a.31703

\begin{abstract}
For tissue engineered bone constructs, calcium phosphate cement (CPC) has a high potential as scaffold material because of its biocompatibility and osteoconductivity. However, in vivo resorption and tissue ingrowth is slow. To address these issues, microspheres can be incorporated into the cement, which will create macroporosity after in situ degradation. The goal of this study was to investigate the handling properties and degradation characteristics of CPC containing gelatin microspheres. Setting time and injectability were determined and an in vitro degradation study was performed. Samples were assayed on mass, compression strength, E-modulus, and morphology. A supplementary degradation test with gelatin microspheres was performed to investigate the influence of physical conditions inside the cement on microsphere stability. The gelatin microsphere CPCs were easy to inject and showed initial setting times of less than $3 \mathrm{~min}$. After 12 -weeks in vitro degradation no increase in macroporosity
\end{abstract}

was observed, which was supported by the small mass loss and stabilizing mechanical strength. Even a clear densification of the composite was observed. Explanations for the lack of macroporosity were recrystallization of the cement onto or inside the gelatin spheres and a delayed degradation of gelatin microspheres inside the scaffold. The supplementary degradation test showed that the $\mathrm{pH}$ is a factor in the delayed gelatin microsphere degradation. Also differences in degradation rate between types of gelatin were observed. Overall, the introduction of gelatin microspheres into CPC renders composites with good handling properties, though the degradation characteristics should be further investigated to generate a macroporous scaffold. (C) 2008 Wiley Periodicals, Inc. J Biomed Mater Res 87A: 643-655, 2008

Key words: calcium phosphate cement; gelatin; microsphere; degradation

\section{INTRODUCTION}

Bone defects caused by trauma or tumor resection and age related loss of bone mass induce a high demand for bone filling materials. Most synthetic alloplasts are not suitable for these types of applications because their mechanical/physical or biological properties are not sufficient, so in most cases autogenous bone is still used. ${ }^{1,2}$ On the other hand, calcium phosphate compounds appear to be an attractive alternative, as they are osteoconductive and can reach to compression strengths that are comparable to

Correspondence to: J. A. Jansen; e-mail: j.jansen@dent. umcn.nl

Contract grant sponsor: Dutch Technology Foundation STW; contract grant number: NGT 6205

(C) 2008 Wiley Periodicals, Inc. bone. ${ }^{3}$ Especially calcium phosphate cement (CPC) is a good candidate since it can be shaped to the defect site after injection and renders an optimal contact between bone and material. ${ }^{4}$ Despite this, the in vivo resorption and tissue ingrowth of most CPCs is slow. ${ }^{5}$ In previous studies ${ }^{6-8}$ poly(DL-lactic-co-glycolic acid) (PLGA) microspheres were added to improve the properties of a CPC for tissue engineering purposes. These microspheres introduced macroporosity into the cement and were also applied for the delivery of growth factors. In vitro degradation tests ${ }^{6}$ showed a slow degradation of the PLGA microspheres that was accompanied by a $\mathrm{pH}$ decrease of the surrounding medium. Compressive strength of the microsphere composites decreased concomitantly as the PLGA eroded, rendering highly macroporous scaffolds.

Next to PLGA other polymers can also be applied for the production of microspheres. Especially natu- 
ral polymers like chitosan, ${ }^{9}$ collagen, ${ }^{10,11}$ and gelatin are interesting candidates that demonstrate enzymatic degradation. Of these polymers, gelatin would be the preferred material because it does not express antigenicity in physiological conditions and is completely resorbable in vivo, unlike collagen. ${ }^{12}$ Gelatin microspheres have been used by different authors for the sustained release of growth factors. ${ }^{13,14}$ Here, the loading of growth factor relies on an ion-binding interaction between an acidic or basic gelatin and a positively/negatively charged protein. ${ }^{15}$ To stabilize gelatin microspheres in an aqueous environment a crosslinker ${ }^{16-18}$ is used. Degradation characteristics of the microspheres can be tailored by changing the crosslinking density. Gelatin can be derived from different animal sources, though porcine (type A, basic) and bovine (type B, acidic) gelatin cover $95 \%$ of both household and industrial use. Food grade (FG) gelatin consists mostly of type A gelatin $(80 \%)$ and less of type B (15\%).

The goal of this study was to characterize the gelatin microsphere CPC on mechanical/ physical properties and degradation characteristics. For this purpose, FG gelatin microspheres with different crosslinking densities were incorporated into the cement. Injectability and setting/cohesive properties of the composites were determined. Furthermore, a 12week in vitro degradation test in proteolytic medium was performed using preset scaffolds. Samples were assayed on mass loss, compressive strength, E-modulus, and morphology. In addition to the in vitro degradation test, a degradation study with gelatin microspheres was performed to investigate the influence of physical conditions inside the cement like $\mathrm{pH}$ and calcium concentration on microsphere stability. Also degradation characteristics of type A and type B gelatin microspheres were compared to the FG gelatin.

\section{MATERIALS AND METHODS}

\section{Materials}

Gelatin (FG, Granules, $\mathrm{pH}=3.8-7.6$, bloom number $=$ 80-120, Merck, Darmstadt, Germany; type A (pI = 7.0-9.0, bloom number $=300) /$ type B $(\mathrm{pI}=4.7-5.2$, bloom number $=225$ ), cell culture tested, Sigma-Aldrich, St. Louis, MO) was used for the preparation of the microspheres. Olive oil (Acros Organics, Geel, Belgium) and acetone (HPLC grade, Labscan, Dublin, Ireland) were used as substrate during microsphere preparation. Glutaraldehyde (25 wt \% solution, EM-grade, Merck, Darmstadt, Germany) was applied as crosslinker. The CPC (Calcibon ${ }^{\circledR}$, Biomet Merck, Darmstadt, Germany) was composed of $61 \% \alpha$-TCP, $26 \%$ $\mathrm{CaHPO}_{4}, 10 \% \mathrm{CaCO}_{3}$, and $3 \%$ precipitated $\mathrm{HA}$. The cement liquid applied was a $1 \%$ aqueous solution of $\mathrm{Na}_{2} \mathrm{HPO}_{4}$.

\section{Methods}

Preparation of gelatin microspheres

$2.5 \mathrm{~g}$ of gelatin was dissolved in $25 \mathrm{~mL}$ demineralized water $\left(\mathrm{ddH}_{2} \mathrm{O}\right)$ for $30 \mathrm{~min}$ at $60^{\circ} \mathrm{C}$. While stirring at 500 rpm using a Teflon upper stirrer, the resulting clear solution was added slowly ( $10 \mathrm{~mL}$ pipette) to a $250-\mathrm{mL}$ threenecked round-bottom flask containing $125 \mathrm{~mL}$ olive oil. During stirring, the round bottom flask was put in an ice bath. After $30 \mathrm{~min}, 50 \mathrm{~mL}$ of chilled acetone $\left(4^{\circ} \mathrm{C}\right)$ and glutaraldehyde $(0.5 \mathrm{~mL}=6.25 \mathrm{mM})$ was added slowly. The solution was stirred for another $1 \mathrm{~h}$ (GEL1) or $2 \mathrm{~h}$ (GEL2) to produce microspheres with a higher extent of crosslinking. Microspheres were collected by filtration (D3, Schott Duran, Mainz, Germany) and washed several times with acetone $(\sim 11)$ to remove residual olive oil. Following this, microspheres were dried over night in a vacuum stove. For the enhanced degradation test all microspheres were crosslinked for $1 \mathrm{~h}$.

\section{Preparation of gelatin microsphere CPC}

Gelatin microspheres were swollen before cement powder or liquid hardener was added to obtain a good distribution of microspheres inside the cement. Therefore, $50 \mathrm{mg}$ of dried GEL1/GEL2 microspheres were put in a 2-mL plastic syringe, subsequently adding $300 \mu \mathrm{L} / 250 \mu \mathrm{L}$ $\mathrm{ddH}_{2} \mathrm{O}$ after which the syringe was stirred vigorously for 15 s using a mixing device (Silamat ${ }^{\circledR}$ mixing apparatus, Vivadent, Schaan, Liechtenstein). Subsequently, $950 \mathrm{mg}$ cement powder was added to the swollen microspheres and the resulting mixture was stirred for another $15 \mathrm{~s}$. Then $300 \mu \mathrm{L}$ of hardener solution $\left(1 \% \mathrm{Na}_{2} \mathrm{HPO}_{4}\right)$ was added and the content was stirred again for $15 \mathrm{~s}$. The resulting cement paste was injected into a Teflon mold (six cylinders, $4.5 \mathrm{~mm} \times 10 \mathrm{~mm}$ ), after which samples were left to set at room temperature for $24 \mathrm{~h}$ and stored in a vacuum stove.

\section{Morphology analysis}

The morphology of the gelatin microspheres and gelatin microsphere CPC was evaluated by scanning electron microscopy (SEM) (JEOL 6400-LINK AN 10000 at $10 \mathrm{kV}$ ). The samples were mounted on aluminum stubs using carbon tape and sputter-coated with gold-palladium before examination.

\section{Size distribution}

The particle size distribution of the gelatin microspheres was determined by image analysis. Dry microspheres were visualized by SEM, while swollen microspheres were characterized with an optical microscope (Leica) after suspension in $\mathrm{ddH}_{2} \mathrm{O}$. The size distribution of both dry and swollen spheres was determined using digital image software (Leica Qwin). 
Injectability

After mixing, the syringe, with an orifice diameter of $1.7 \mathrm{~mm}$, was fitted vertically in a fixture and put under the platen of a tensile bench set (858 MiniBionixII ${ }^{\mathbb{R}}$, MTS Corp., Eden Prairie, MN) in tension mode. ${ }^{6}$ The cement composites were compressed until $\sim 1 \mathrm{cc}$ was present inside the syringes, which was used as starting point. Twenty-five seconds after mixing the cement (for $15 \mathrm{~s}$ ), a compressive force was applied to the syringe with a constant velocity of $10 \mathrm{~mm} / \mathrm{min}$. The compressive force was recorded as a function of the plunger travel length ${ }^{19}$ after which it was converted into time. The time from mixing the paste until pressure reaches $60 \mathrm{~N}$, is referred to as the injection time. If all the paste is injected before pressure increase, the paste is regarded as fully injectable. All tests were performed threefold $(n=3)$.

Setting time

The initial and final setting times were assessed using custom available Gillmore needles (ASTM C266). A bronze block was used as mold containing six holes $(6 \mathrm{~mm}$ in diameter, $12 \mathrm{~mm}$ in height). The mold was placed in a water bath to simulate body temperature $\left(37^{\circ} \mathrm{C}\right)$. Samples were mixed and injected into the mold in a retrograde fashion, after which the initial and final setting time of the samples was determined $(n=3)$.

For determination of the cohesive properties of the gelatin microsphere CPC, samples were injected into Ringer's solution at $37^{\circ} \mathrm{C}$. During the cement setting time it was observed whether the paste retained its original configuration or powder formation/disintegration occurred. Every anomaly was recorded.

\section{Porosity}

The macro- and total-porosity of preset gelatin microsphere CPC samples was determined. The macroporosity is the porosity in which the pores are created by the degradation of gelatin microspheres. The total porosity is the macroporosity plus the original microporosity of the cement. When the gelatin microspheres are not degraded, the macroporosity correspond to the vol \% of microspheres initially present inside the cement.

To measure these parameters, both gelatin microsphere CPC samples and microporous CPC samples of a known volume were placed in an oven at $650^{\circ} \mathrm{C}$ for $2 \mathrm{~h}$. After burning out the gelatin/moisture, samples were weighed and Eqs. (1) and (2) were used for the derivation of the total porosity and the macroporosity. Tests were performed threefold $(n=3)$.

$$
\begin{gathered}
\varepsilon_{\text {tot }}=\left(1-\frac{m_{\text {macro } / \text { micro }}}{V \times \rho_{\mathrm{HAP}}}\right) \times 100 \% \\
\varepsilon_{\text {macro }}=\left(1-\frac{m_{\text {macro }}}{m_{\text {micro }}}\right) \times 100 \%
\end{gathered}
$$

where $\varepsilon_{\text {tot }}=$ total porosity $(\%), \varepsilon_{\text {macro }}=$ macroporosity $(\%), m_{\text {macro }}=$ average mass macroporous sample (after burning out gelatin) $(\mathrm{g}, n=3), m_{\text {micro }}=$ average mass microporous sample $(\mathrm{g}, n=3), V=$ volume sample $\left(\mathrm{cm}^{3}\right)$, $\rho_{\text {HAP }}=$ density hydroxy apatite $\left(\mathrm{g} / \mathrm{cm}^{3}\right)$.

\section{Degradation assay}

For the degradation assay gelatin microsphere CPC samples were prepared as described earlier. Samples were placed in $3 \mathrm{~mL}$ of phosphate-buffered saline (PBS, pH 7.4) containing $50 \mu \mathrm{g} / \mathrm{mL}$ gentamycin and $373 \mathrm{ng} / \mathrm{mL}$ collagenase $1 \mathrm{~A}(\text { Sigma })^{13}$ and incubated at $37^{\circ} \mathrm{C}$ in a water bath on a shaker table $(70 \mathrm{rpm})$ for 12 weeks. Every 3-4 days sample buffer was refreshed. At days 1, 3, 7, 14, 28, 42, and 84, specimens were subjected to analysis according to the descriptions summarized later. Samples were taken fivefold $(n=5)$. Gelatin microspheres (8-9 mg, dry spheres) and microporous cement scaffolds were used as a control.

\section{pH measurements}

After removal of the samples from the water bath the $\mathrm{pH}$ of the PBS medium was measured.

\section{Mass loss quantification}

Samples were freeze-dried overnight before measuring the mass. The mass loss of the samples was calculated using Eq. (3).

$$
R_{L}=\frac{M_{0}-M_{n}}{M_{0}} \times 100 \%
$$

where $R_{\mathrm{L}}=$ mass loss sample at $t=n(\%), M_{0}=$ Mass sample at $t=0(\mathrm{~g}), M_{n}=$ Mass sample at $t=n(\mathrm{~g})$.

\section{Morphology}

The morphology of the samples was determined using SEM. To visualize composite degradation and increase in (macro)porosity, overview pictures were taken at a magnification of $\times 100$. Also, pictures were taken at higher magnifications to visualize the microsphere degradation. Next to that, energy dispersive X-ray spectrometry (EDS) was performed for further material analysis.

\section{Mechanical characteristics}

Samples were placed in a mechanical testing bench $(858$ MiniBionixII ${ }^{\circledR}$, MTS Corp., Eden Prairie, MN) and both compressive strength and E-modulus along the height of the specimens were measured at a crosshead speed of $0.5 \mathrm{~mm} / \mathrm{min}$.

\section{Supplementary microsphere degradation study}

In addition to the 12-weeks degradation study, a degradation study using gelatin microspheres was performed to investigate the influence of physical conditions inside the cement like $\mathrm{pH}$ and calcium concentration on microsphere 

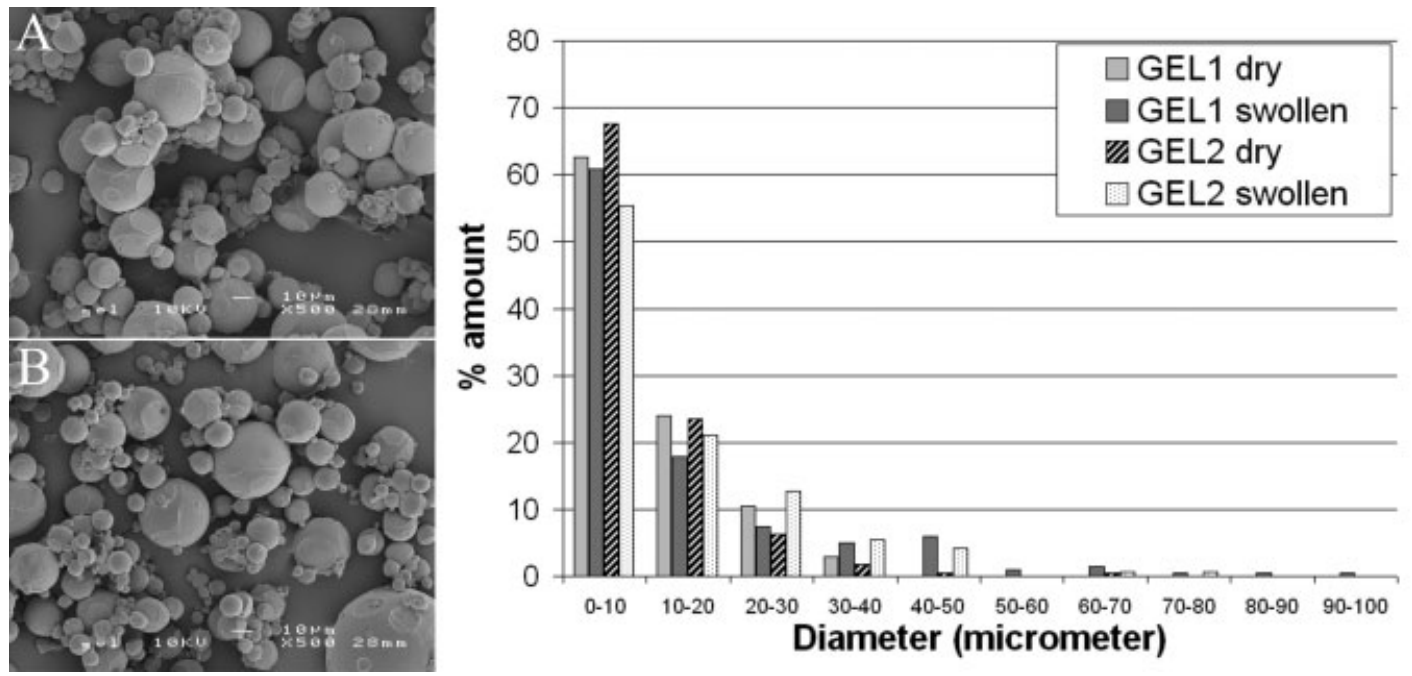

C

Figure 1. SEM images of the morphology of GEL1 microspheres (A), GEL2 microspheres (B), and size distribution of dry and swollen microspheres (C), group size: $n=171 / 166$ (dry microspheres), $n=201 / 222$ (swollen microspheres).

stability. Also, the degradation characteristics of FG gelatin microspheres were compared to microspheres of type A and type B gelatin. In this study the degradation of the microspheres was enhanced by applying a high concentration of gelatin-degrading enzyme, which enabled us to compare the degradation characteristics of these microspheres within a short time period. For this purpose $10 \mathrm{mg}$ of $1 \mathrm{~h}$ crosslinked gelatin microspheres of either type $\mathrm{A}, \mathrm{B}$, or FG were put in $3 \mathrm{~mL}$ PBS containing $10 \mu \mathrm{g} / \mathrm{mL}$ collagenase $1 \mathrm{~A}$ at $37^{\circ} \mathrm{C}$. Applying these conditions, a pilot study showed that FG microspheres were fully degraded within 3 days. Because an exponential degradation profile was observed in this pilot, a time period of 1 day was chosen for the degradation study. To mimic the conditions inside the gelatin microsphere CPCs, gelatin microspheres were pretreated for 1 day in PBS containing $0.1 \mathrm{M} \mathrm{CaCl}_{2}(\mathrm{pH}=5.72)$ before they were exposed to the enhanced degradation medium. PBS ( $\mathrm{pH}=7.4)$ and acidic PBS $(\mathrm{pH}=5.72)$ were taken as a control. Samples were assayed on mass loss and morphology $(n=3)$.

\section{Statistical analysis}

Data were arranged as mean \pm standard deviation. Significant differences were determined using analysis of variance (ANOVA). Results were considered significant if $p<$ 0.05 . Calculations were performed using GraphPad Instat ${ }^{\circledR}$.

\section{RESULTS}

\section{Preparation of microspheres}

SEM-micrographs of the prepared microspheres are shown in Figure 1(A,B). Dry microspheres were partially agglomerated and imprints of the interconnections between the spheres were visible at the surface. In an aqueous environment, the GEL1 and GEL2 microspheres showed $\sim 1.5$ times increase in diameter (Table I) during which the interconnections were broken. The size distribution [Fig. 1(C)] showed that most unswollen microspheres were below $10 \mu \mathrm{m}$ in diameter and an increase in sphere size higher than $30 \mu \mathrm{m}$ was observed after swelling. Overall, a slightly higher percentage of larger spheres was observed in the GEL1 group.

\section{Preparation and clinical handling properties of gelatin microsphere CPC}

A SEM-micrograph of the microspheres inside the cement is given in Figure 2 (A), which is representative for both GEL1 and GEL2 microsphere CPCs. A

TABLE I

Physical Parameters Cement/Microsphere Formulations

\begin{tabular}{lccc}
\hline & Microporous $^{6}$ & GEL1 CPC & GEL2 CPC \\
\hline Average microsphere size $(\mu \mathrm{m})$ & - & 9.70 (dry) & 8.64 (dry) \\
& & 14.79 (swollen) & 13.76 (swollen) \\
Initial setting time $(\mathrm{s})$ & $108.3 \pm 5.8$ & $165.0 \pm 15.0$ & $145.0 \pm 8.7$ \\
Injection time $(\mathrm{s})$ & 108 & 128 & 120 \\
Mass $(\mathrm{mg})$ & $262.7 \pm 6.1$ & $166.0 \pm 5.3$ & $174.2 \pm 5.5$ \\
Porosity $^{\mathrm{a}}$ & $45.52 \pm 1.26$ & $69.67 \pm 1.29$ & $69.21 \pm 0.36$ \\
Macro porosity $^{\mathrm{a}}$ & - & 44.32 & 43.49 \\
\hline
\end{tabular}

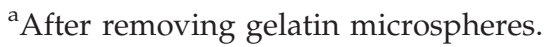



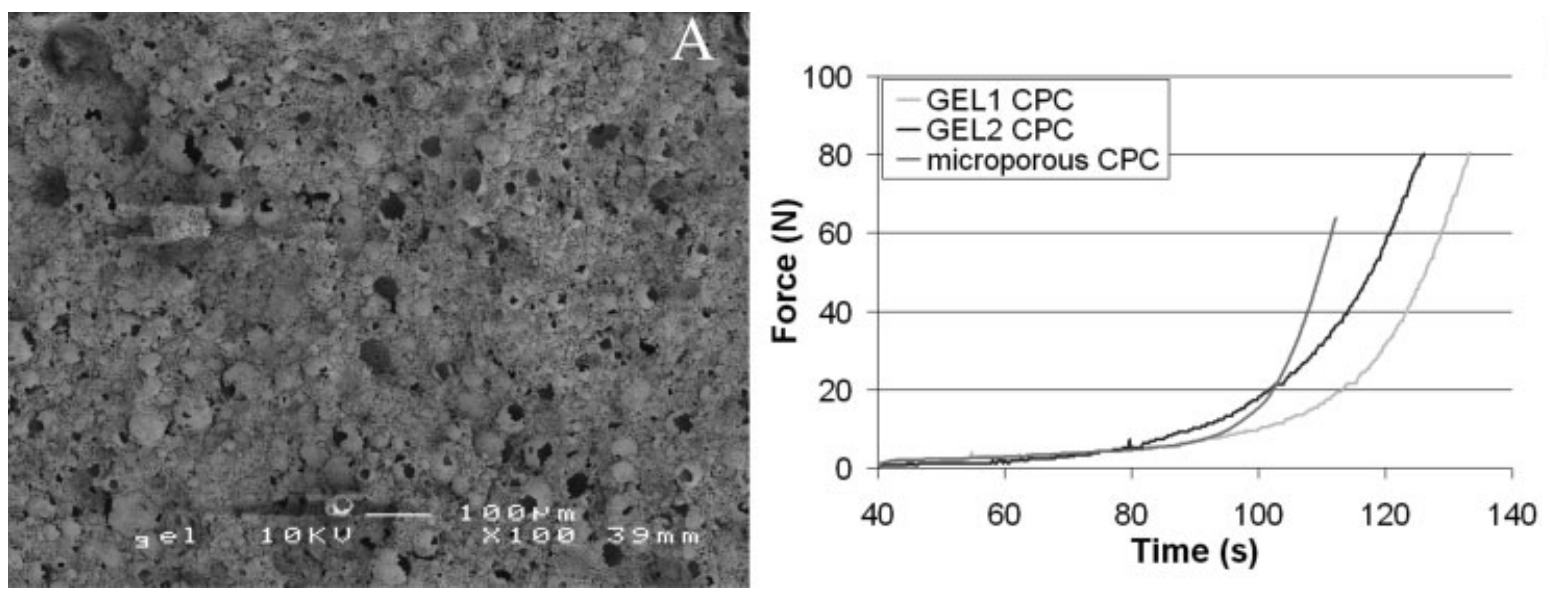

Figure 2. Morphology of gelatin microsphere CPC (A) and injectability graph of GEL1/GEL2 microsphere CPC and microporous $\mathrm{CPC}^{6}(\mathrm{~B})$.

substantial percentage of the spheres was broken, showing an eggshell structure. Furthermore, an equal distribution of microspheres inside the cement was obtained.

The injectability graph of the gelatin microsphere CPCs [Fig. 2(B)] showed that the pressure on the syringe increased at a later time point than the microporous cement, ${ }^{6}$ indicating that the composite cement was easier to inject. When comparing both gelatin microsphere CPCs, the GEL1 composite showed a longer injection time, that is, better injectability than the GEL2 composite. The setting time (Table I) of both gelatin microsphere CPCs was comparable to each other and slightly higher than the microporous cement $(p<0.01)$. No final setting time was measured due to the high water content of the gelatin microspheres. Cohesion tests revealed no powder formation or disintegration when injecting the samples in an aqueous environment. The macroporosity of both GEL1 and GEL2 microsphere CPCs was 43$44 \%$, with a total porosity of $69-70 \%$.

\section{Degradation assay}

In Figure 3 (A) the cumulative $\mathrm{pH}$ of the PBS medium is given as a function of the degradation time. The medium of both gelatin microsphere CPCs showed an initial $\mathrm{pH}$ increase, followed by a decrease and stabilized at a $\mathrm{pH}$ of 6.1 at $t=40$ days. The release medium of both GEL1 and GEL2 microspheres showed a small decrease at a $\mathrm{pH}$ of 7.1.

The development of the mass in time is shown in Figure 3(B). For both gelatin microsphere CPCs an initial increase of $2 \%$ was followed by a slow decrease in mass. After 12 weeks a maximum decrease of $2 \%$ was observed. The microporous cement only showed an increase in mass of 3\% that appeared to decrease after 12 weeks. Significant differences in mass loss between the microporous cement and gelatin microsphere CPCs were observed ( $p<0.05$ at $t=4,8$ weeks), though only the GEL1 microsphere CPC showed a significant decrease in mass at $t=12$ weeks $(p<0.01)$. The gelatin microspheres showed an initial increase in mass that was higher than the increase of the gelatin microsphere CPCs, but decreased very rapidly after 3 days. Significant differences between the GEL1 and GEL2 microspheres were observed at day 1 and 14 $(p<0.05)$, although the trend was similar. The development of the compression strength of the gelatin microsphere CPCs over time is given in Figure 4 (A). An initial increase was observed over 7 days, after which the compression strength remained almost constant for 12 weeks. The final compression strength of both gelatin microsphere CPCs was $30 \mathrm{MPa}$, which was significantly lower than the microporous cement $(p<0.001)$. The E-modulus [Fig. 4(B)] showed a comparable trend with respect to the compression strength with an increase till day 7 after which it remained stable at $\pm 2000 \mathrm{MPa}$. Also here the microporous cement was significantly higher $(p<0.05)$ and no significant differences were observed between the GEL1 and GEL2 composites.

The morphology of the gelatin microsphere CPCs and gelatin microspheres separately are given in Figures 5 and 6 . For both gelatin microsphere CPCs small differences in morphology were observed between samples from day 1 to week 12. Microsphere structures were still visible after 12 weeks and no increase in macroporosity was observed during the degradation test. In contrast, comparison of samples taken at day 1 with samples taken at later time points showed even a clear densification of the material. At higher magnifications (Fig. 7), the presence of crystals onto/inside the gelatin microspheres 

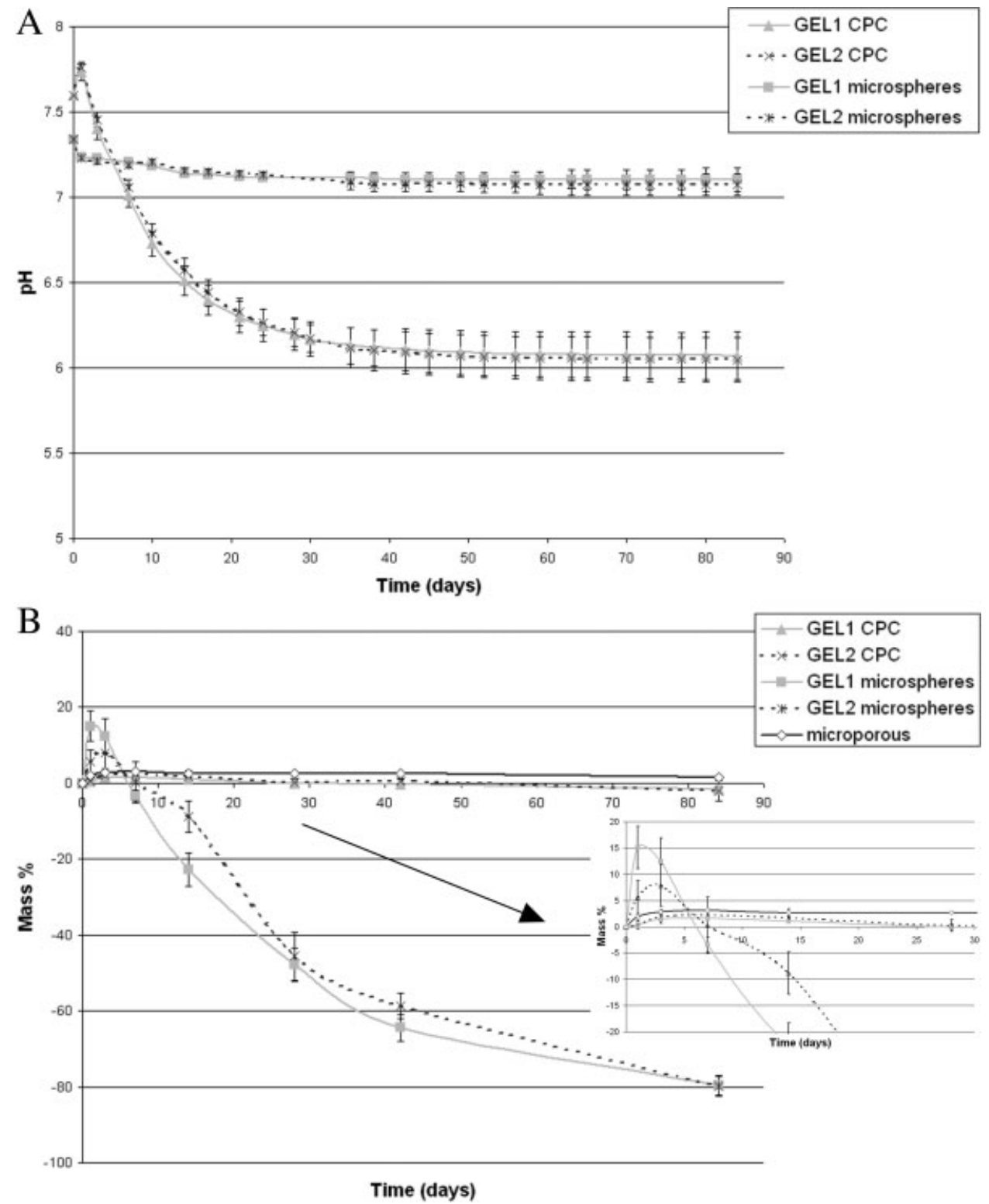

Figure 3. $\mathrm{pH}$ decrease of surrounding medium (A) and mass loss (B) as a function of degradation time of the GEL1/ GEL2 microsphere CPC, microspheres, and microporous cement.

was observed. EDS analysis revealed that these crystals consisted of calcium and phosphate which indicated a recrystallization of the cement at these sites.

The separate gelatin microspheres showed already deformations at day 1 , slowly losing their spherical shape and fusing into a network of gelatin particles at week 4 and 6 . After 12 weeks no separate gelatin microspheres were distinguished anymore. The structures still visible with SEM were traced to accumulations of PBS salts and some salts remaining from the gelatin. Differences between the GEL1 and GEL 2 microspheres were visible especially at 4 weeks where the remaining structures of the GEL2 microspheres showed a smaller, more particle-like morphology.

\section{Supplementary microsphere degradation study}

Figure 8 shows the $\%$ mass left after 1 day in degradation medium using three types of gelatin and three different pretreatment methods. The results showed that the pretreatment method was important since both PBS $+\mathrm{CaCl}_{2}$ and PBS $(\mathrm{pH}=5.72)$ groups degraded significantly slower than the PBS $(\mathrm{pH}=7.4)$ group with all types of gelatin (Table II). A difference between the PBS $(\mathrm{pH}=5.72)$ group and the PBS + $\mathrm{CaCl}_{2}$ group was observed with type $\mathrm{B}$ gelatin that exhibited a higher mass loss with the PBS $+\mathrm{CaCl}_{2}$ group. Furthermore it was observed that both type $\mathrm{A}$ and type $B$ gelatin degraded significantly faster than the FG gelatin within all pretreatment groups. 

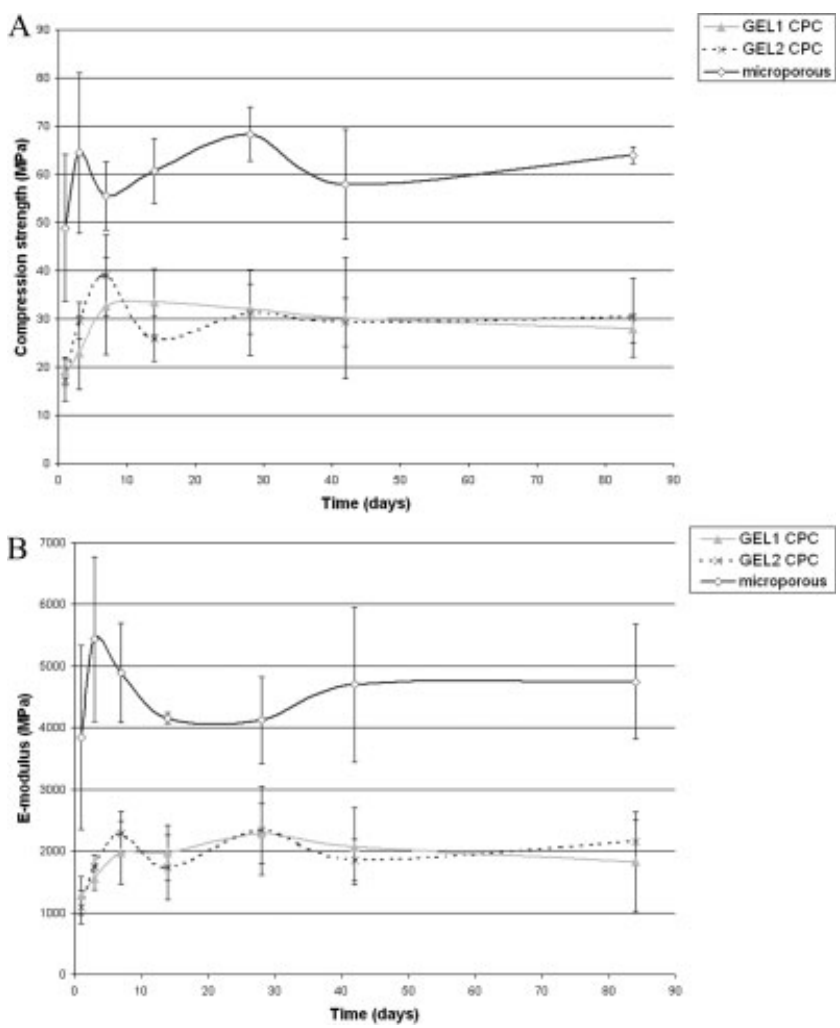

Figure 4. Compression strength (A) and E-modulus (B) as a function of degradation time of the GEL1/GEL2 microsphere CPC and microporous cement.

Figures 9-11 depict SEM-micrographs of gelatin type A, type B, and FG microspheres before and after pretreatment and after 1 day in enhanced degradation medium. Before pretreatment only perfect spheres were present, after pretreatment the spheres were deformed within all groups. After 1 day in enhanced degradation medium, microparticle structure was lost in the PBS ( $\mathrm{pH}=7.4)$ groups, while with the PBS $+\mathrm{CaCl}_{2}$ and $\mathrm{PBS}(\mathrm{pH}=5.72)$ groups microparticles or a structure of elongated/connected microparticle structures were still visible. Also, morphological degradation was found to differ between the various types of gelatin. Microspheres prepared of FG gelatin maintained their microsphere structure very well, while type A gelatin microspheres showed deformations into an agglomerate structure within all pretreatment groups. Type B gelatin was intermediate, with the PBS $+\mathrm{CaCl}_{2}$-group showing a separate microparticle structure and the PBS $(\mathrm{pH}$ $=5.72$ ) group showing an agglomerate structure consisting of thin, elongated microparticles.

\section{DISCUSSION}

In this study gelatin microspheres were added to a microporous CPC to investigate the possibility of these composite materials to serve as an injectable bone substitute, and secondly as a possible creator of macroporosity after gelatin degradation. For this purpose, FG gelatin microspheres with different crosslinking density were added to the cement and subjected to physical/chemical characterization. An in vitro degradation study with these composites was performed in proteolytic degradation medium to see whether macroporosity could be created. Furthermore, to investigate the influence of physical conditions inside the cement like $\mathrm{pH}$ and calcium concentration on microsphere stability, a supplementary degradation study was performed. In this degradation study also microspheres of cell-culturetested-gelatin type A and type B were investigated and compared to the FG gelatin microspheres.

The physical characteristics of the cement with added gelatin microspheres differed significantly from the microporous cement. Initial setting time showed a significant increase and final setting time
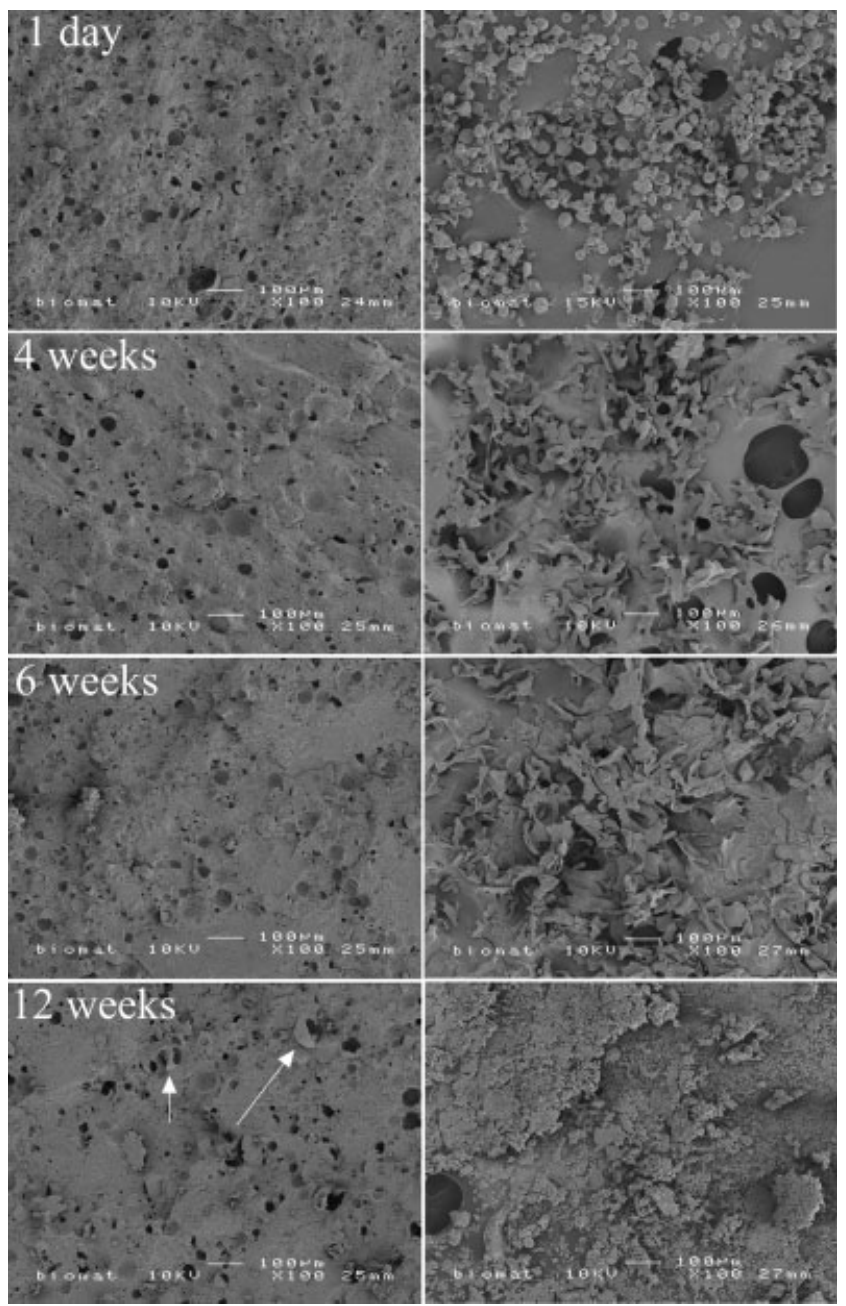

Figure 5. SEM-micrographs of GEL1 microsphere CPC (left) and GEL1 microspheres (right) at $t=1$ day and 4, 6, and 12 weeks (original magnification $\times 100$ ), arrows indicate the presence of gelatin microsphere shells. 


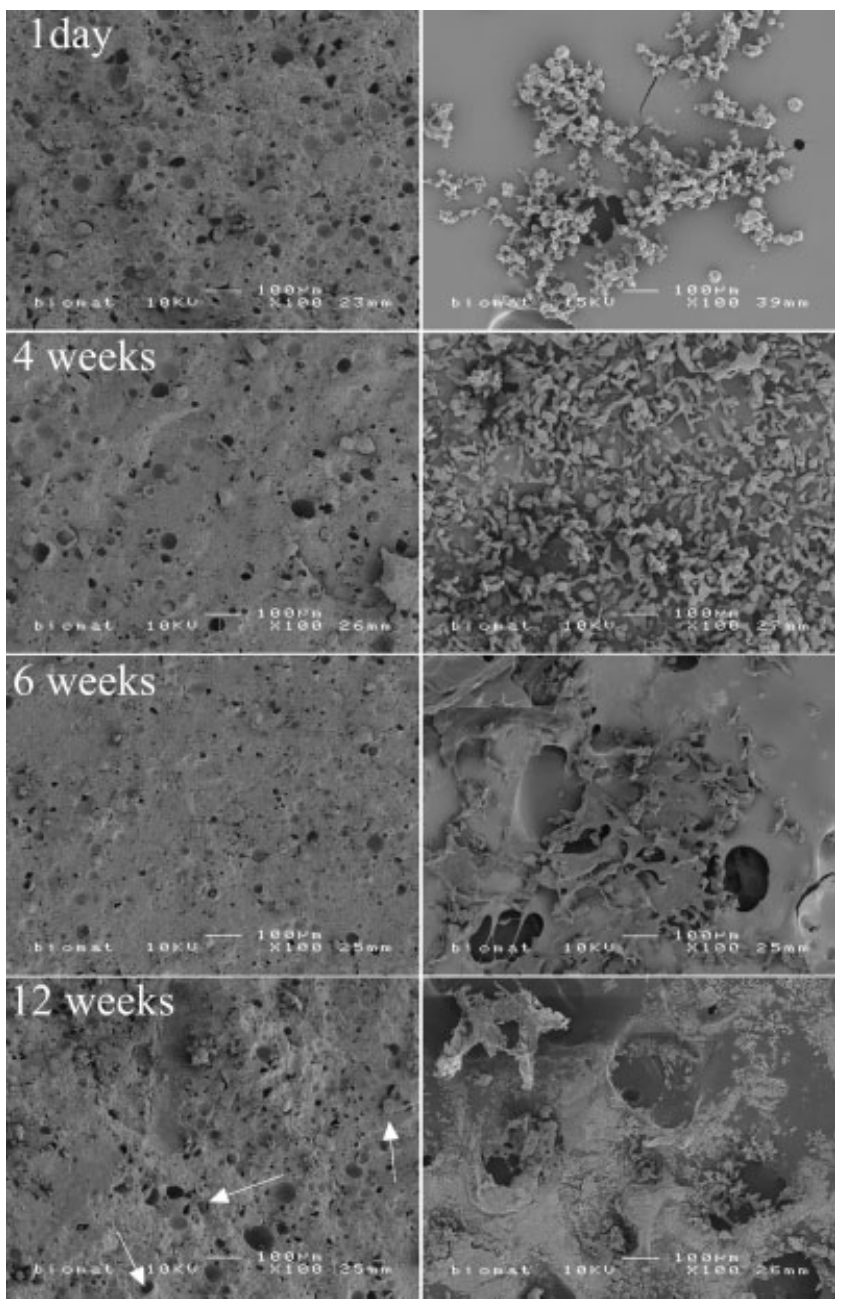

Figure 6. SEM-micrographs of GEL2 microsphere CPC (left) and GEL2 microspheres (right) at $t=1$ day and 4, 6, and 12 weeks (original magnification $\times 100$ ), arrows indicate the presence of gelatin microsphere shells.

was not measurable. An explanation for this is that the gelatin microspheres were added to the cement in a swollen state to obtain a good distribution. The water inside these microspheres contributed to cement setting, leading to increased setting times. ${ }^{3}$ On the other hand, the addition of gelatin microspheres to the cement resulted in improved injection properties, as injection time increased for the gelatin microsphere CPC when compared to microporous cement. Differences in injectability were observed between the GEL1 and GEL2 composite where increased crosslinking of the gelatin microspheres led to more stiffer structures. ${ }^{20}$ After cement setting, SEM revealed that the microspheres inside the gelatin microsphere CPCs showed a structure of broken, hollow spheres. It can be hypothesized that physical bonds between the gelatin microspheres and surrounding cement were responsible for this structure as shrink stresses, arisen during drying, forced the immobilized spheres to break.

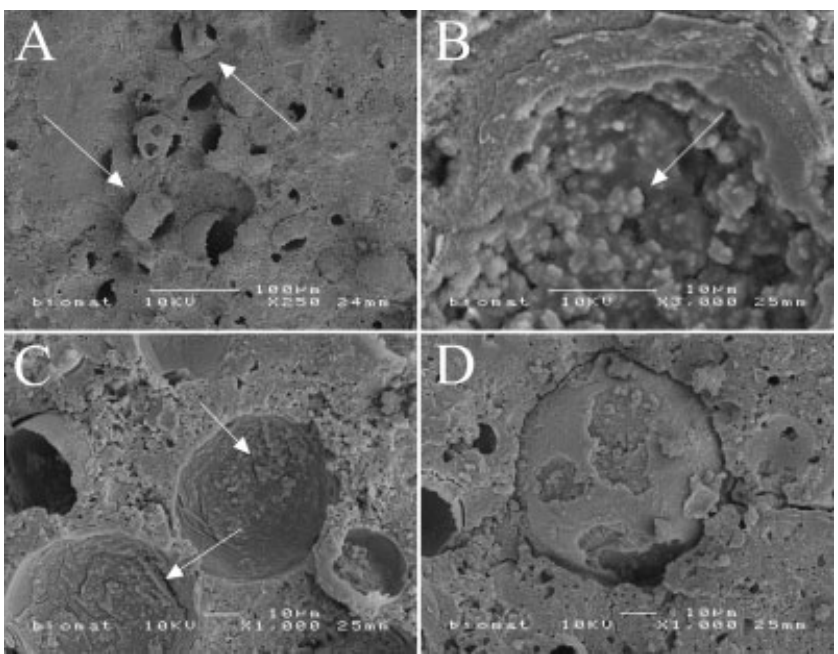

Figure 7. Close-up pictures of gelatin microspheres inside the cement, showing precipitate onto/inside the gelatin microspheres (arrows); (A) GEL1 3 days (original magnification $\times 250$ ), (B) GEL1 2 weeks (original magnification $\times$ 3000), (C) GEL1 6 weeks (original magnification $\times 1000$ ), (D) GEL2 6 weeks (original magnification $\times 1000$ ).

The degradation experiment showed an initial increase in mass for the gelatin microsphere CPCs at day 1 and 3 . This increase in mass was also observed in a higher extent with the separate gelatin microspheres and the microporous cement. An explanation is the precipitation of PBS crystals on the outside of the cement samples and microspheres during the experiment. The high increase observed with separate gelatin microspheres was caused by a similar mechanism. Here, weight was measured after gently removing the medium above the microsphere gel and freeze-drying the microspheres. Because the swollen gelatin microspheres contained about six times their wt \% of water, they also retained a PBS precipitate that contributed to the weight of the samples. Rinsing the microspheres with demineralized water before drying is not a solution, since this will

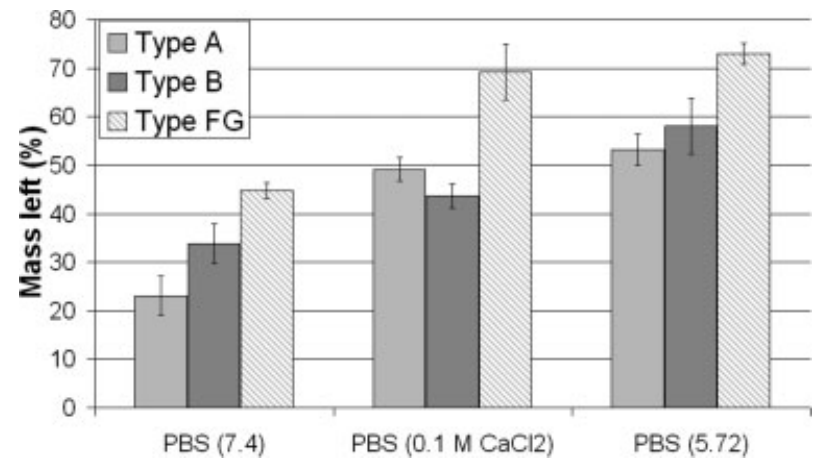

Figure 8. Mass remaining at $t=1$ day of enhanced degradation with Type $\mathrm{A}$, Type $\mathrm{B}$, and FG gelatin microspheres after pretreatment in PBS with $\mathrm{pH}=7.4,0.1 \mathrm{M}$ $\mathrm{CaCl}_{2}$ or $\mathrm{pH}=5.72$. 
TABLE II

Statistical Significance Samples Supplementary Degradation Study

\begin{tabular}{|c|c|c|}
\hline Parameter & Samples & $\overline{\text { Significance }}$ \\
\hline \multirow[t]{7}{*}{ Pretreatment method } & Type A PBS (7.4) vs. type A PBS $+\mathrm{CaCl}_{2}$ & $p<0.001$ \\
\hline & Type A PBS (7.4) vs. type A PBS (5.72) & $p<0.001$ \\
\hline & Type B PBS (7.4) vs. type B PBS $+\mathrm{CaCl}_{2}$ & $p<0.05$ \\
\hline & Type B PBS (7.4) vs. type B PBS (5.72) & $p<0.001$ \\
\hline & Type B PBS $+\mathrm{CaCl}_{2}$ vs. type B PBS (5.72) & $p<0.001$ \\
\hline & Type FG PBS (7.4) vs. type FG PBS $+\mathrm{CaCl}_{2}$ & $p<0.001$ \\
\hline & Type FG PBS (7.4) vs. type FG PBS (5.72) & $p<0.001$ \\
\hline \multirow[t]{7}{*}{ Type of gelatin } & Type A PBS (7.4) vs. type B PBS (7.4) & $p<0.05$ \\
\hline & Type A PBS (7.4) vs. type FG PBS (7.4) & $p<0.001$ \\
\hline & Type B PBS (7.4) vs. type FG PBS (7.4) & $p<0.01$ \\
\hline & Type A PBS $+\mathrm{CaCl}_{2}$ vs. type FG PBS $+\mathrm{CaCl}_{2}$ & $p<0.001$ \\
\hline & Type B PBS $+\mathrm{CaCl}_{2}$ vs. type FG PBS $+\mathrm{CaCl}_{2}$ & $p<0.001$ \\
\hline & Type A PBS (5.72) vs. type FG PBS (5.72) & $p<0.001$ \\
\hline & Type B PBS (5.72) vs. type FG PBS (5.72) & $p<0.001$ \\
\hline
\end{tabular}

greatly influence the mass of the microspheres themselves as degradation products dissolve during rinsing and microspheres will get lost.

The initial decrease in $\mathrm{pH}$ was also observed in a previous experiment ${ }^{6}$ and is due to the release of acidic products from the cement, while the microspheres themselves only show a minimal decrease in $\mathrm{pH}$.

The gelatin microsphere CPCs also showed a striking increase in mechanical strength within the first 7 days. This increase is due to the recrystallization of the cement into hydroxyapatite (HA), which is welldescribed in literature. ${ }^{3}$ In addition, the clear densification of the cement observed with SEM is also most likely caused by cement recrystallization. Because of the biomimetic properties of the gelatin, ${ }^{12}$ the fractured microspheres showed a shell of calcium phosphate $(\mathrm{CaP})$ precipitate onto the surface forming a strong bond between the cement and the gelatin.

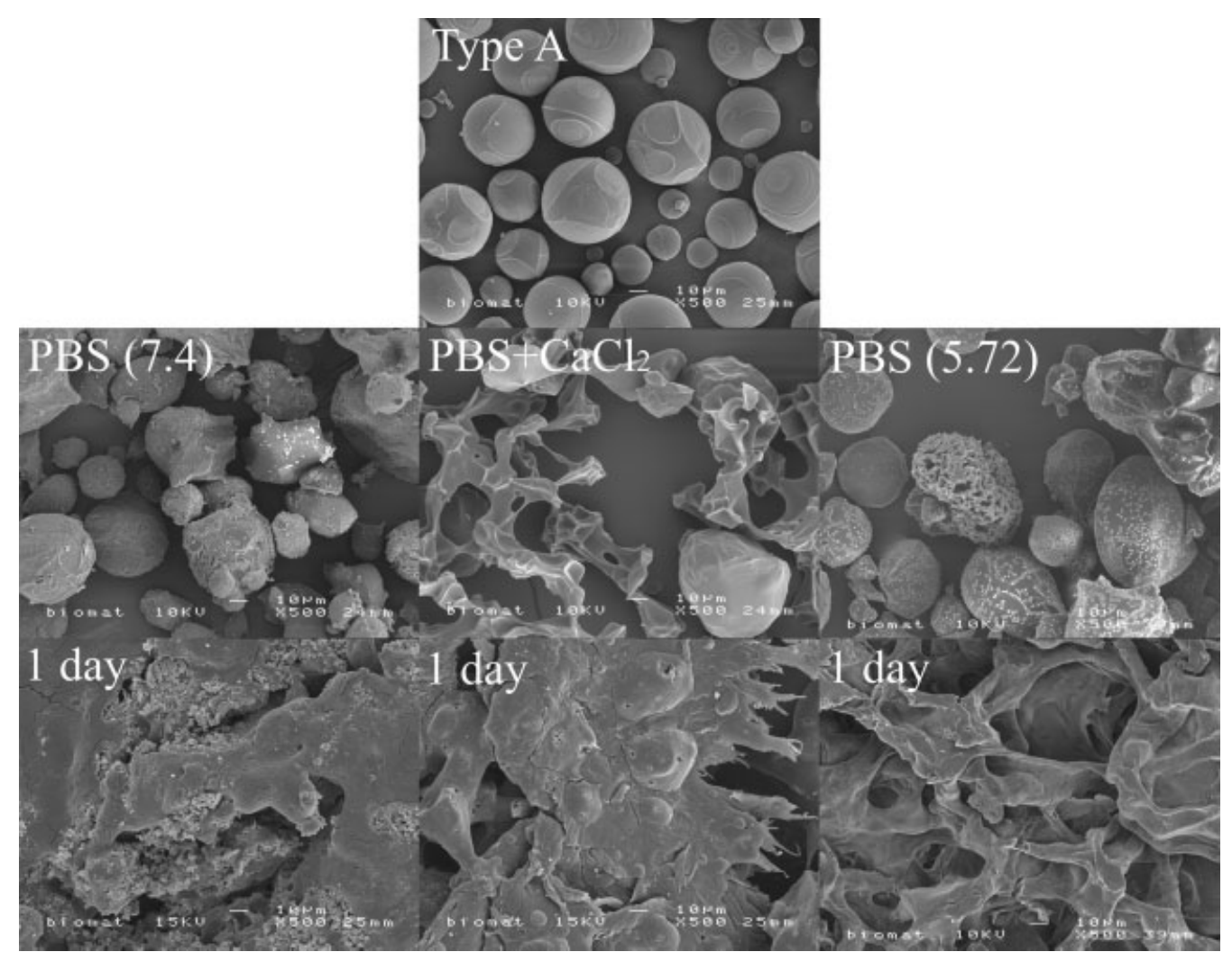

Figure 9. SEM-micrographs of Type A gelatin microspheres before pretreatment (upper), after pretreatment in PBS with $\mathrm{pH}=7.4,0.1 \mathrm{M} \mathrm{CaCl}_{2}$ or $\mathrm{pH}=5.72$ (middle), and after $t=1$ day of enhanced degradation study (lower) (original magnification $\times 500)$. 


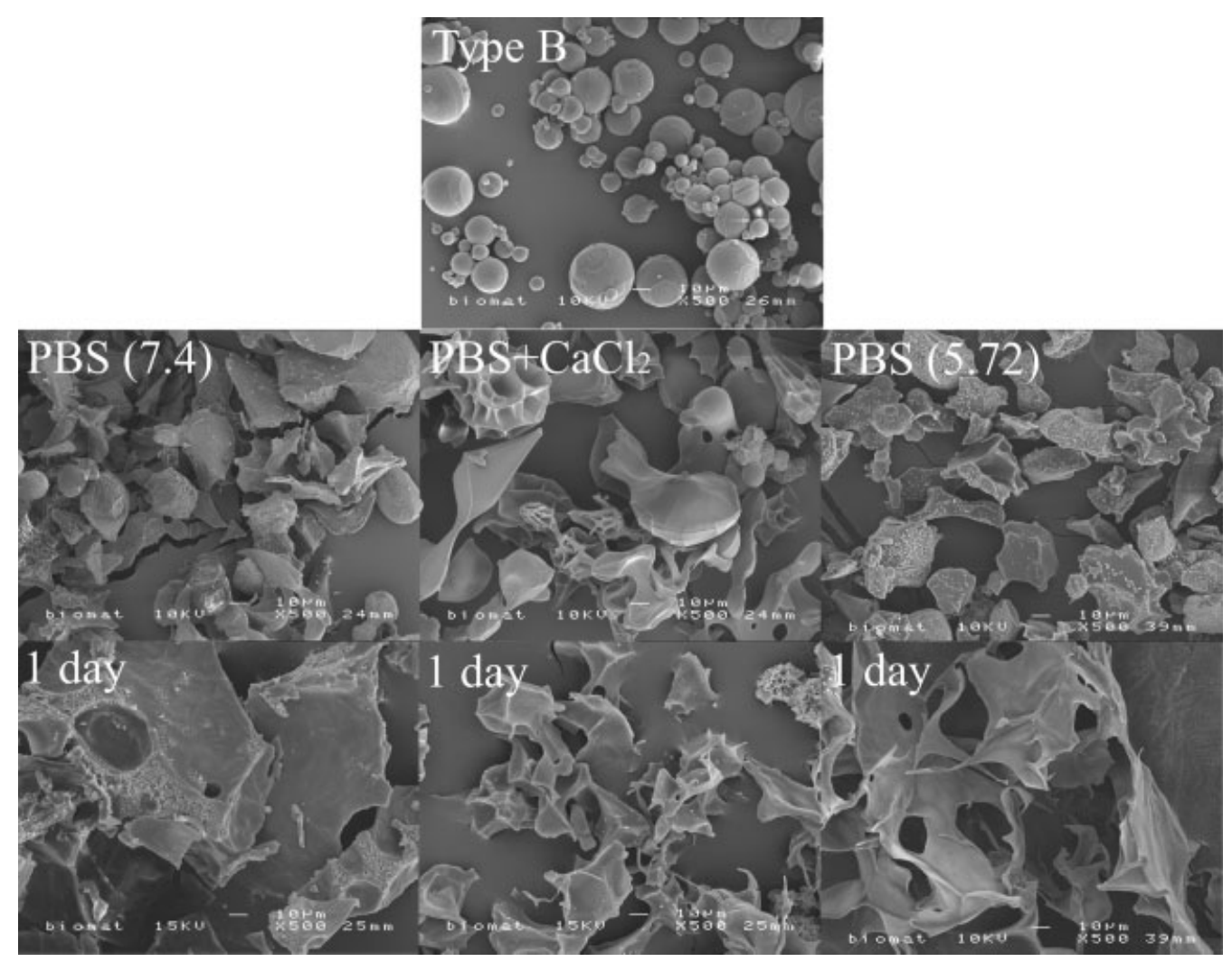

Figure 10. SEM-micrographs of Type B gelatin microspheres before pretreatment (upper), after pretreatment in PBS with $\mathrm{pH}=7.4,0.1 \mathrm{M} \mathrm{CaCl}_{2}$ or $\mathrm{pH}=5.72$ (middle), and after $t=1$ day of enhanced degradation study (lower) (original magnification $\times 500)$.

Comparison of the current results with PLGA microsphere CPCs in a previous experiment, ${ }^{6}$ the PLGA microspheres were always present without a shell of $\mathrm{CaP}$ precipitate. At later time points ( $>2$ weeks) even the formation of crystals inside the gelatin microspheres was observed, indicating that the pores formed by the microspheres can be filled with a precipitate.

At the end of the degradation test, the obtained scaffold did not show an increase in macroporosity or increased cement resorption. A first explanation for these results is the occurrence of the discussed $\mathrm{CaP}$ precipitates onto and inside gelatin microspheres. The vol \% of gelatin microspheres inside the composite should be sufficient to form interconnections between the microspheres. However, if $\mathrm{CaP}$ is precipitating between two contacting microspheres, no interconnection will be formed but a shell of crystalline HA. Increasing the \% of microspheres inside the cement, can solve this problem partially as the influence of the cement will be smaller and more interconnections will be present. On the other hand, precipitation will still occur and the addition of more than $5 \mathrm{wt} \%$ of microspheres greatly increases setting time and decreases mechanical properties. ${ }^{3,6}$
Secondly, the weight loss of the GEL1 and GEL2 microsphere CPCs was very small when compared to the microporous cement and did not exceed the $5 \mathrm{wt} \%$ of microspheres added initially. If the gelatin microspheres inside the cement composite had degraded as fast as the separate gelatin microspheres, the composites should have shown a higher decrease in mass and distinct morphological degradation of the microspheres after 6-12 weeks. SEMpictures at these time points still showed the presence of intact gelatin shells throughout the composite and therefore it can be concluded that the microspheres degraded slower inside these composites. An explanation for the delayed degradation is binding/deactivation of the enzyme to the CPC. Since gelatin degrades by an enzymatic route, the calcium phosphate matrix of the gelatin microsphere CPC can prevent the enzyme from entering the material. Collagenase is prone to bind to $\mathrm{CPC}$, which was proven to have a protein-binding capacity. ${ }^{7,21,22}$ According to Kremer et al. ${ }^{23}$ a suspension of HA crystals in combination with free $\mathrm{Ca}^{2+}$-ions $(>0.5 \mathrm{mM})$ can even induce an autolytic degradation of matrix metalloprotease-1 (MMP-1, interstitial collagenase). A decrease in $\mathrm{pH}$, as observed with the gelatin microsphere CPCs, also could have influenced collagenase 


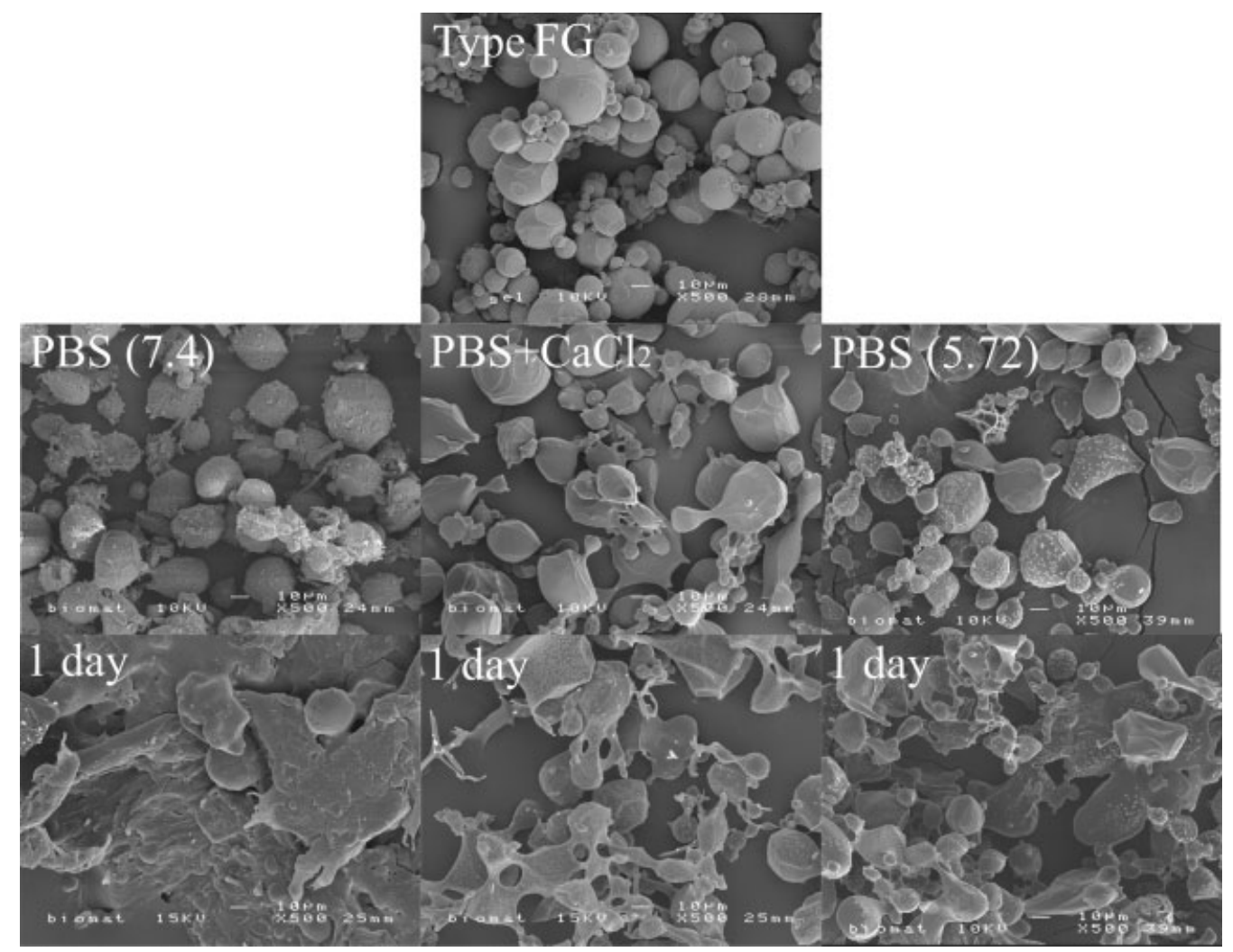

Figure 11. SEM-micrographs of Type FG gelatin microspheres before pretreatment (upper), after pretreatment in PBS with $\mathrm{pH}=7.4,0.1 \mathrm{M} \mathrm{CaCl}$ or $\mathrm{pH}=5.72$ (middle), and after $t=1$ day of enhanced degradation study (lower) (original magnification $\times 500)$.

activity. However, the optimal $\mathrm{pH}$ range for collagenase is $6.3-8^{24}$ and individual $\mathrm{pH}$ values measured during the test never dropped below 6.8.

From the supplementary microsphere degradation test it can be concluded that a small $\mathrm{pH}$ decrease as is present inside the microporous cement, ${ }^{6}$ delays gelatin microsphere degradation by changing the microsphere structure/stability. This is most likely caused by the $\mathrm{pH}$-dependent crosslink reaction of residual aldehyde groups after subjecting the microspheres/composite to the degradation medium. In this experiment gelatin microspheres were crosslinked with glutaraldehyde to ensure structural stability of the spheres during cement mixing. The amount of glutaraldehyde used $(6.25 \mathrm{mM})$ and crosslink time ( $1 \mathrm{~h}$ minimum) were just sufficient for sphere stability because a fast degrading microsphere was proposed to give a superior cement composite. However, despite this low amount of crosslinker, with the applied crosslink method free aldehyde groups were still present after microsphere preparation as glutaraldehyde partially exists as oligomers with multiple aldehyde chains. ${ }^{25}$ In aqueous conditions, for example the degradation medium, these aldehyde chains can further react with free amino groups, preferentially lysine or arginine in the gelatin ${ }^{26}$ which leads to the formation of new crosslinks, in particularly Schiff bases $\left(\mathrm{R}_{1} \mathrm{R}_{2} \mathrm{C}=\mathrm{N}-\mathrm{R}_{3}\right.$, with $\mathrm{R}_{3}=$ alkylic or arylic). ${ }^{27,28}$ As aldehyde groups become more electrophilic at a lower $\mathrm{pH}^{29}$ the modest $\mathrm{pH}$ decrease must have accelerated these crosslink reactions thereby stabilizing the microspheres against proteolytic degradation. An approach to prevent this delayed crosslink reaction is for example the addition of an aldehyde reagent (inhibitor) to the oil phase at the end of microsphere preparation. Gly$\operatorname{cin}^{13,30}$ is commonly used as it is a body-own substance. Also, the combined used of citric acid and citric acic/glycin ${ }^{31}$ shows inhibition of the crosslink reaction.

Next to covalent bonding of gelatin chains, noncovalent bonding also played a small role in the gelatin microsphere CPC degradation as was observed in the supplementary degradation test. $\mathrm{Ca}^{2+}$-ions are present inside the degradation medium due to the dissolution of monetite and calcium carbonate. These $\mathrm{Ca}^{2+}$ ions can bind to multiple sites in proteins, ${ }^{32}$ especially electrostatic interactions with the carboxy groups ${ }^{33}$ from amino acids, like aspartate and glutamate, ${ }^{26}$ can give stable bonds (Fig. 12). An example of such a mechanism is given by Reinhardt et al. ${ }^{34}$ who observed that $\mathrm{Ca}^{2+}$ binding to motifs in Fibrillin-1 (an extracellular matrix protein) stabilizes the structure against proteolytic degradation. In contrast 


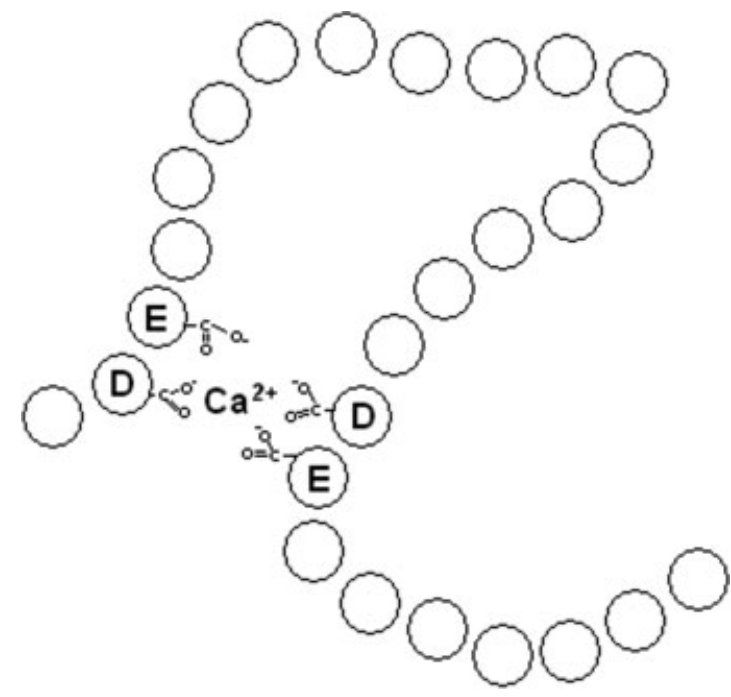

Figure 12. Folding of protein chains by interaction with $\mathrm{Ca}^{2+}$.

to this result, in this study the addition of $\mathrm{Ca}^{2+}$-ions led to an increase in degradation rate as was observed with type $B$ gelatin microspheres. Here, the microspheres of the PBS $+\mathrm{CaCl}_{2}$ group showed a significant higher weight loss than the PBS $(\mathrm{pH}=$ 5.72) group, whereas the $\mathrm{pH}$ during pretreatment of both groups was the same. An explanation is that with type B gelatin $\mathrm{Ca}^{2+}$ crosslinks existed that stabilized the microparticle morphology but did not hamper microsphere degradation. The maintained microparticle structure possibly enhanced proteolytic degradation because of the larger surface area of these structures. The higher amount of carboxylic groups of type B gelatin (100-115 mM) when compared to type A gelatin $(78-80 \mathrm{mM})^{35}$ was responsible for the difference between the gelatin types, giving type $\mathrm{B}$ gelatin a better $\mathrm{Ca}^{2+}$-binding capacity. Regarding this capacity, another explanation for the higher degradation rate is a difference in enzyme activity. Because $\mathrm{Ca}^{2+}$ serves as a cofactor for interstitial collagenase, ${ }^{36,37}$ different authors have shown a reactivation of collagenase/gelatinase in the presence of 3.7-10 $\mathrm{mM}$ of free $\mathrm{Ca}^{2+} \cdot{ }^{38,39}$ Therefore if the gelatin also released $\mathrm{Ca}^{2+}$ during proteolytic degradation it reactivated the enzyme, which would be more pronounced with the type B gelatin.

Comparison of the three types of gelatin in the supplementary degradation test revealed that in all pretreatment groups type $\mathrm{A}$ and type $\mathrm{B}$ gelatin microspheres degraded significantly faster than FG gelatin microspheres. The main difference between the two cell-culture tested gelatins and the FG gelatin is the bloom number that is significantly lower with the FG gelatin. The bloom number is a measure of gel strength of the gelatin ${ }^{35}$ and is proportional to the average molecular weight of the chains. This implies that FG gelatin has a significant lower molecular weight and produces more gelatin chains per unit of microsphere surface than type A or type B gelatin. Therefore it can be hypothesized that with type $\mathrm{A}$ and type $\mathrm{B}$ gelatin microspheres, enzymatic cleavage of a single "large" gelatin chain has a higher impact on microsphere degradation than with the FG gelatin, which results in a faster degradation.

\section{CONCLUSION}

The prepared gelatin microsphere CPCs were easy to handle with an initial setting time of less than 3 min. They possessed a compression strength of \pm $30 \mathrm{MPa}$ and a microsphere content of $43-44 \mathrm{vol} \%$. In vitro degradation showed a modest $\mathrm{pH}$ decrease, a mechanical strength that remained constant in time and a small decrease in mass. SEM-investigation of the gelatin microsphere CPCs during the degradation test revealed little change in morphology, where separate gelatin microspheres showed complete degradation within the same time period. At the end of the degradation test, no increase in macroporosity was observed but even a clear densification of the composite. Parameters that contributed to this phenomenon were cement recrystallization inside/onto the microspheres and a delayed gelatin degradation. From the supplementary microsphere degradation test it was concluded that the modest $\mathrm{pH}$ decrease present inside the cement is a factor in the delayed gelatin degradation. Also differences in degradation rate between different types of gelatin were observed. Overall, the gelatin microsphere CPC showed good handling properties, though degradation characteristics should be further investigated to generate a macroporous scaffold.

The authors would like to thank T.A. Holland and Z.S. Patel for technical assistance. Scanning electron microscopy was performed at the Microscopic Imaging Centre (MIC) of the Nijmegen Centre for Molecular Life Sciences (NCMLS), the Netherlands.

\section{References}

1. Goldberg VM, Stevenson S. Natural history of autografts and allografts. Clin Orthop Relat Res 1987;225:7-16.

2. Imola MJ, Sciarretta V, Schramm VL. Skull base reconstruction. Curr Opin Otolaryngol Head Neck Surg 2003;11:282290.

3. Khairoun I, Boltong MG, Driessens FCM, Planell JA. Effect of calcium carbonate on clinical compliance of apatitic calcium phosphate bone cement. J Biomed Mater Res B Appl Biomater 1997;38:356-360.

4. Jansen JA, Ooms E, Verdonschot N, Wolke JGC. Injectable calcium phosphate cement for bone repair and implant fixation. Orthop Clin North Am 2005;36:89-95. 
5. Ooms EM, Wolke JGC, van der Waerden JPCM, Jansen JA. Trabecular bone response to injectable calcium phosphate (Ca-P) cement. J Biomed Mater Res 2002;61:9-18.

6. Habraken WJEM, Wolke JGC, Mikos AG, Jansen JA. Injectable PLGA microsphere/calcium phosphate cements: Physical properties and degradation characteristics. J Biomater Sci Polym Ed 2006;17:1057-1074.

7. Ruhé $P Q$, Hedberg EL, Torio Paron N, Spauwen PHM, Jansen JA, Mikos AG. RhBMP-2 release from injectable poly (DL-lactic-co-glycolic acid)/calcium-phosphate cement composites. J Bone Joint Surg Am 2003;85:75-81.

8. Ruhé PQ, Boerman OC, Russel FG, Spauwen PH, Mikos AG, Jansen JA. Controlled release of rhBMP-2 loaded poly(DLlactic-co-glycolic acid)/calcium phosphate cement composites in vivo. J Control Release 2005;106:162-171.

9. Muzzarelli C, Muzzarelli RAA. Natural and artificial chitosan-inorganic composites. J Inorg Biochem 2002;92:89-94.

10. Berthold A, Cremer K, Kreuter J. Collagen microparticles: Carriers for glucocorticosteroids. Eur J Pharm Biopharm 1998;45:23-29.

11. Swatschek D, Schatton W, Muller W, Kreuter J. Microparticles derived from marine sponge collagen (SCMPs): Preparation, characterization and suitability for dermal delivery of all-trans retinol. Eur J Pharm Biopharm 2002;54:125-133.

12. Bigi A, Boanini E, Panzavolta S, Roveri N, Rubini K. Bonelike apatite growth on hydroxyapatite-gelatin sponges from simulated body fluid. J Biomed Mater Res 2002;59:709-714.

13. Holland TA, Tabata Y, Mikos AG. Dual growth factor delivery from degradable oligo(poly(ethylene glycol) fumarate) hydrogel scaffolds for cartilage tissue engineering. J Control Release 2005;101:111-125.

14. Bruschi ML, Cardoso MLC, Lucchesi MB, Gremião MPD. Gelatin microparticles containing propolis obtained by spraydrying technique: Preparation and characterization. Int J Pharm 2003;264:45-55.

15. Yamamoto M, Ikada Y, Tabata Y. Controlled release of growth factors based on biodegradation of gelatin hydrogel. J Biomater Sci Polym Ed 2001;12:77-88.

16. Muvaffak A, Gurhan I, Hasirci N. Prolonged cytotoxic effect of colchicine released from biodegradable microspheres. J Biomed Mater Res B Appl Biomater 2004;71:295-304.

17. Vandelli MA, Rivasi F, Guerra P, Forni F, Arletti R. Gelatin microspheres crosslinked with D,L-glyceraldehyde as a potential drug delivery system: Preparation, characterisation, in vitro and in vivo studies. Int J Pharm 2001;215:175-184.

18. Liang H-C, Chang W-H, Lin K-J, Sung H-W. Genipin-crosslinked gelatin microspheres as a drug carrier for intramuscular administration: In vitro and in vivo studies. J Biomed Mat Res A 2003;65:271-282.

19. Khairoun I, Magne D, Gauthier O, Bouler JM, Aguado E, Daculsi G, Weiss P. In vitro characterization and in vivo properties of a carbonated apatite bone cement. J Biomed Mater Res 2002;60:633-642.

20. Bigi A, Cojazzi G, Panzavolta S, Rubini K, Roveri N. Mechanical and thermal properties of gelatin films at different degrees of glutaraldehyde crosslinking. Biomaterials 2001;22:763-768.

21. Stallmann HP, Faber C, Slotema ET, Lyaruu DM, Bronckers ALJJ, Nieuw Amerongen AV, Wuisman PIJM. Continuous- release or burst-release of the antimicrobial peptide human lactoferrin 1-11 (hLF1-11) from calcium phosphate bone substitutes. J Antimicrob Chemother 2003;52:853-855.

22. Ziegler J, Mayr-Wohlfart U, Kessler S, Breitig D, Günther K-P. Adsorption and release properties of growth factors from biodegradable implants. J Biomed Mater Res 2002;59:422-428.

23. Kremer EA, Chen Y, Suzuki K, Nagase H, Gorski JP. Hydroxyapatite induces autolytic degradation and inactivation of matrix metalloprotease-1 and -3. J Bone Miner Res 1998; 13:1890-1902.

24. Schomberg D, Salzmann M, editors. Enzyme Handbook. Berlin: Springer-Verlag; 1991.

25. Monsan P, Puzo G, Mazarguil H. Etude du mechanisme d'etablissement des liaisons glutaraldehyde-proteines. Biochimie 1975;57:1281-1292.

26. Tristam GR. The Proteins. New York: Academic Press; 1953.

27. Simionescu A, Sinionescu D, Deac R. Lysine-enhanced glutaraldehyde crosslinking of collagenous biomaterials. J Biomed Mater Res 1991;25:1494-1505.

28. Molin S-O, Nygren H, Dolonius L. A new method for the study of glutaraldehyde-induced crosslinking properties in proteins with special reference to the reaction with amino groups. J Histochem Cytochem 1978;26:412-414.

29. Morrison RT, Boyd RN. Organic Chemistry, Vol. 3. Boston: Allyn and Bacon; 1973. p 641-643.

30. Matsuda S, Iwata H, Naomi S, Ikada Y. Bioadhesion of gelatin films crosslinked with glutaraldehyde. J Biomed Mater Res 1999;45:20-27.

31. Singh S, Rama Rao KV, Venugopal K, Manikandan R. Alteration in dissolution characteristics of gelatin-containing formulations: A review of the problem, test methods, and solutions. Pharm Technol 2002;26:36-58.

32. Yamashita MM, Wesson L, Eisenman G, Eisenberg D. Where metal ions bind in proteins. Proc Natl Acad Sci USA 1990;87: 5648-5652.

33. Peng $\mathrm{S}, \mathrm{Wu} \mathrm{C}$. $\mathrm{Ca}^{2+}$-induced complexation between thermally sensitive spherical poly( $\mathrm{N}$-vinyl-caprolactam-co-sodium acrylate) microgels and linear gelatin chains in water. Polymer 2001;42:7343-7347.

34. Reinhardt DP, Ono RN, Sakai LY. Calcium stabilizes fibrillin1 against proteolytic degradation. J Biol Chem 1997;272:12311236.

35. Standard methods for the sampling and testing of gelatins. Gelatin Manifacturers Institue of America, 505 fifth Ave., Room 1015, New York, NY.

36. Morgunova E, Tuuttila A, Bergmann U, Isupov M, Lindquist Y, Schneider G, Tryggvason K. Structure of human pro-matrix metalloproteinase-2: Activation mechanism revealed. Science 1999;284:1667-1670.

37. Borkakoti N. Matrix metalloprotease inhibitors: Design from structure. Biochem Soc Trans 2004;32:17-20.

38. Winberg J-O, Berg E, Kolset SO, Uhlin-Hansen L. Calciuminduced activation and truncation of promatrix metalloprotease- 9 linked to the core protein of chondroitin sulfate proteoglycans. Eur J Biochem 2003;270:3996-4007.

39. Sharpe C, Robinson JJ. Characterization of matrix metalloprotease activities induced in the sea urchin extraembryonic matrix, the hyaline layer. Biochem Cell Biol 2001;79:461-468. 